\title{
Attosecond Time Delay in Photoemission and Electron Scattering near Threshold
}

\author{
Anatoli S. Kheifets and Alexander W. Bray \\ Research School of Physics and Engineering, The Australian National University, \\ Canberra, Australian Capital Territory 2601, Australia
}

\author{
Igor Bray \\ Curtin Institute for Computation and Department of Physics, Astronomy and Medical Radiation Science, \\ Curtin University, Perth, Western Australia 6845, Australia
}

(Received 21 July 2016; published 28 September 2016)

\begin{abstract}
We study the time delay in the primary photoemission channel near the opening of an additional channel and compare it with the Wigner time delay in elastic scattering of the photoelectron near the corresponding inelastic threshold. The photoemission time delay near threshold is significantly enhanced, to a measurable 40 as, in comparison to the corresponding elastic scattering delay. The enhancement is due to the different lowest order of interelectron interaction coupling the primary and additional photoemission channels. We illustrate these findings by considering photodetachment from the $\mathrm{H}^{-}$negative ion, and compare it with electron scattering on the hydrogen atom near the first excitation threshold. Other threshold processes of atomic photoionization and molecular photofragmentation, where photoemission time delay is enhanced, are identified. This opens the possibility of studying threshold behavior utilizing attosecond chronoscopy.
\end{abstract}

DOI: 10.1103/PhysRevLett.117.143202

Collisions near threshold play a special role in quantum physics by providing an essential link between weakly bound states and unbound continuous waves. Aggregation or fragmentation of quantum particles always proceed via thresholds. Sufficiently close to the threshold, the de Broglie wavelength of these particles is large compared to their interaction length. This allows for a very general analytical description leading to universal threshold laws $[1,2]$. A variety of atomic species such as electrons, positrons, ions, and cold atoms are available for high-precision tests of these laws [3]. In solids, these threshold laws are modified in low-dimensional systems such as quantum dots [4].

Attosecond time delay in atomic photoemission can provide an alternative route for observing threshold effects. Analytic properties of the scattering amplitude near threshold are defined by the scattering phases. Because time delay is intimately linked to the phase, it can be a very useful tool for studying these analytic properties. The opening of a new channel corresponds to a branching point of the scattering amplitude in the complex energy plane because the number of physically possible quantum states of the system changes. Phase determination near such a branching point brings significantly richer information in comparison to any other regular energy point [5].

The first experimental observations of time delay in photoemission [6,7] gave rise to the rapidly developing field of attosecond chronoscopy, which opened a new perspective on ultrafast electron dynamics [8]. The time delay in photoemission is interpreted in terms of the Wigner time delay introduced for particle scattering in external potential [9-11]. It is a delay, or advance, of a particle traveling through a potential landscape in comparison with the same particle traveling in a free space. The Wigner time delay is calculated as an energy derivative of the scattering phase in a given partial wave. A similar definition is adopted in photoemission, where the time delay is related to the photoelectron group delay, and evaluated as an energy derivative of the phase of the complex ionization amplitude (see, e.g., [6,12]).

In this Letter, we study time delay in the primary photoemission channel near the opening of an additional channel and compare it with the Wigner time delay in the elastic scattering of the photoelectron near the corresponding inelastic threshold. We show that the photoemission time delay near threshold is significantly larger than the corresponding elastic scattering delay because of the different lowest order of interelectron interaction coupling the primary and additional photoemission channels. We illustrate these findings by considering photodetachment from the $\mathrm{H}^{-}$negative ion and comparing it with electron scattering on the hydrogen atom near the first excitation threshold. Both processes are strongly affected by interelectron interaction. However, the lowest order of this interaction is considerably different. Elastic electron scattering on the ground state of the hydrogen atom contains excitation to the $n=2$ state in the second order of interelectron interaction. Alternatively, photodetachment from $\mathrm{H}^{-}$to the ground state of $\mathrm{H}$ needs just one such interaction because of the significant fraction of the $2 s^{2}$ configuration in the ground state of $\mathrm{H}^{-}$. Above the $n=2$ excitation threshold, it appears that both the photodetachment and elastic scattering amplitude experience rapid growth of their phases. However, this growth is an order of magnitude faster in photodetachment in comparison with 
photoelectron scattering. This results in an order of magnitude enhancement of the photoemission time delay near threshold.

We define the photoemission time delay as

$$
\tau=(d / d E) \arg f(E) \equiv \operatorname{Im}\left[f^{\prime}(E) / f(E)\right] .
$$

The complex photoionization (or photodetachment) amplitude is given by the partial wave expansion

$$
\begin{aligned}
f(E)= & (2 \pi)^{3 / 2} k^{-1 / 2} \sum_{\substack{l=l_{i} \pm 1 \\
m=m_{i}}} e^{i \delta_{l}(E)} i^{-l} Y_{l m}(\hat{k}) \\
& \times\left(\begin{array}{rrr}
l & 1 & l_{i} \\
m & 0 & m_{i}
\end{array}\right)\left\langle k l\|D\| n_{i} l_{i}\right\rangle .
\end{aligned}
$$

Here, $\left\langle k l\|D\| n_{i} l_{i}\right\rangle$ is the reduced dipole matrix element stripped of all the angular momentum projections. The light is polarized linearly along the quantization axis $z$. The partial photoionization cross section for the transition from an occupied state $n_{i} l_{i}$ to the photoelectron continuum state $k l$ is calculated as

$$
\sigma_{n_{i} l_{i} \rightarrow k l}(\omega)=(4 / 3) \pi^{2} \alpha \omega\left|\left\langle k l\|D\| n_{i} l_{i}\right\rangle\right|^{2},
$$

with $\omega$ being the photon energy and $\alpha$ the fine structure constant. Atomic units $e=m=\hbar=1$ are used in this expression and throughout the Letter. The atomic unit of time is 24.2 as, where 1 as $=10^{-18} \mathrm{~s}$.

Our numerical results, both for electron scattering and photodetachment, are obtained within the convergent closecoupling (CCC) formalism. This formalism is well tested both for the $e-\mathrm{H}$ scattering [13] and photodetachment of $\mathrm{H}^{-}$[14]. The CCC formalism [15] has been adapted for photoionization calculations of two-electron targets ( $\mathrm{He}$ and its isoelectronic sequence [14] and alkaline-earth metal atoms [16]) as well as three-electron targets ( $\mathrm{Li}$ [17]). In the two-electron CCC formalism, the reduced dipole matrix element entering Eq. (2) is written as [18]

$$
\begin{aligned}
\left\langle k \ln _{i} l_{i}\|D\| \Psi_{0}\right\rangle= & \left\langle k \ln _{i} l_{i}\|d\| \Psi_{0}\right\rangle+\sum_{l_{j} n_{j}} \sum_{l^{\prime}} \sum_{k^{\prime}} \\
& \times \frac{\left\langle k l l_{i} n_{i}\left\|T_{J S}\right\| n_{j} l_{j} k^{\prime} l^{\prime}\right\rangle}{E-k^{\prime 2} / 2-\epsilon_{j}+i 0}\left\langle l^{\prime} k^{\prime} l_{j} n_{j}\|d\| \Psi_{0}\right\rangle .
\end{aligned}
$$

Here, $J=1$ and $S=0$ are the total orbital momentum and spin of the electron pair, respectively, and $E=k^{2} / 2+\epsilon_{i}$ is the final state energy. The uncorrelated dipole matrix element $\|d\|$ is calculated with a 20 -term Hylleraas expansion $\Psi_{0}$ as in the previous study [14].

The two terms on the right-hand side of Eq. (4) are represented graphically in Fig. 1. Here, the solid line with an arrow to the right (the time direction) indicates an

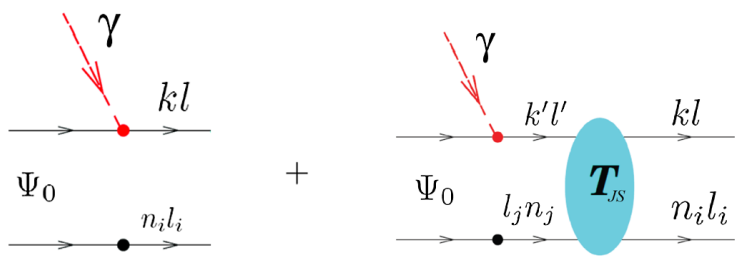

FIG. 1. Graphical representation of the two-electron photoionization amplitude in the CCC formalism.

electron, the dotted line exhibits a photon and a shaded oval represents the half-off-shell $T_{J S}$ matrix which describes the electron-hydrogen scattering away from the energy shell.

This is found through solution of the LippmannSchwinder integral equations as part of the CCC calculations (see [13] for more detail). The on-shell component of the $T$ matrix [Eq. (38) of [19]] determines the scattering $S$ matrix and the phase shift $\phi_{l}(k)$ relative to the Coulomb phase $\sigma_{l}(k)$ in the given partial wave

$$
\begin{aligned}
& \phi_{l}(k)=\sigma_{l}(k)+\arg S_{l}(k) / 2, \\
& S_{l}(k)=1-2 \pi i k\left\langle k l l_{i} n_{i}\left\|T_{J S}\right\| n_{i} l_{i} k l\right\rangle .
\end{aligned}
$$

Results of the CCC calculation for $\mathrm{H}^{-}$are shown near threshold in Fig. 2 and compared with an analogous calculation for He on an extended energy range in Fig. 3. Both targets are left in the ground $1 s$ state of the residual core, i.e., $n_{i}=1$ and $l_{i}=0$. In the $\mathrm{H}^{-}$calculation, we use a CCC $(20,2)$ model, which indicates summation over $0 \leq l_{j} \leq 2$ and $1 \leq n_{j} \leq 20-l_{j}$ in Eq. (4). The discrete states with $n_{j} \geq 5$ have positive energy and represent the ionization continuum. It is in this way that double photoionization is included in the CCC model. The He calculation was performed with a $\operatorname{CCC}(15,3)$ model. Both calculations were performed with a 20-term Hylleraas expansion and were essentially gauge insensitive. The $V$-gauge results are shown in Figs. 2 and 3.

In the top panel of Fig. 2, we show the photodetachment cross section in comparison with several sets of experimental data collated in [21] and the latest measured set reported in [22]. The CCC calculation demonstrates a good agreement with the experiment except for the photoelectron energy range below $0.1 \mathrm{eV}$ for which the older experimental data sets [21] are lower. A more recent calculation [20] lends support to the CCC result. In the middle panel, we plot the phase shift (5) obtained from the scattering $S$ matrix. The analogous phase shift extracted from the dipole $D$ matrix (2) is indistinguishable on the scale of the figure except in the vicinity of the $n=2$ threshold at $E_{t}=3 / 4 \mathrm{Ry}$ $(10.2 \mathrm{eV})$. In the same panel, we show results of a variational calculation [23] and a hyperspherical closecoupling calculation [24] for the $e-\mathrm{H}$ scattering phase in the dipole singlet channel. All the phase calculations are in good agreement. The bottom panel shows the time delay 

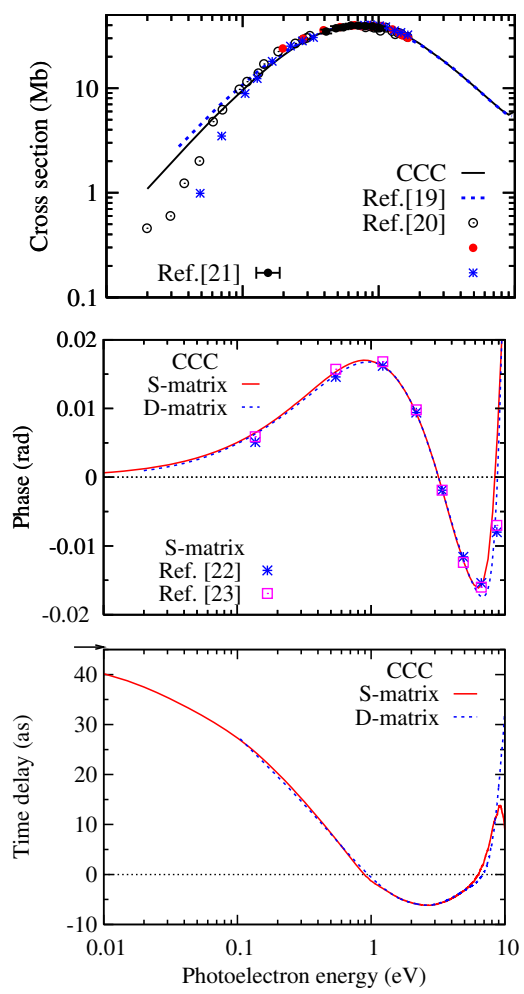

FIG. 2. Top: photodetachment cross section. The CCC calculation (black solid line) is compared with a $B$-spline calculation [20] (blue dashed line) and the experimental data collated in [21] (the original plotting symbols are used). The black points with error bars represent the experimental data of [22]. Middle: phase shift extracted from the scattering $S$ matrix (6) (red solid line) and the photoemission amplitude (1) ( $D$ matrix, blue dashed line). The literature values [23] and [24] for $e-\mathrm{H}$ scattering in the singlet dipole channel are plotted with asterisks and open squares, respectively. Bottom: the energy derivative of the $S$ - and $D$-matrix phases from the CCC calculations are converted to the time delay. The arrow indicates the asymptotic limit of zero photoelectron energy.

calculated from the energy derivative of the phase shift of the $S$ matrix (5) and the $D$ matrix using Eq. (1).

The scattering phase shift in a short range potential should follow the Wigner threshold law $\delta_{l} \propto E^{l+1 / 2}$ [27]. This would entail $\delta_{p} \propto E^{3 / 2}$ in the partial $p$ wave which defines the photoemission time delay from a spherically symmetric target like $\mathrm{H}^{-}$. Therefore, the time delay should be vanishing at the threshold as $\tau \propto E^{1 / 2}$. This is, indeed, the case for the phase returned by a Hartree-Fock (HF) calculation in the frozen core approximation [28]. The polarization correction changes this result dramatically. For a polarization potential $V(r)=\beta / r^{4}$, the scattering phase is $\delta_{p}(E)=(2 \pi \beta / 15) E$, and the time delay becomes a constant $\tau_{p}(E)=(2 \pi \beta / 15)$ [see Eq. (26) of [3]]. Comparing this expression with the numerical $\mathrm{CCC}$ result $\tau_{p}\left(\mathrm{E}=10^{-5} \mathrm{eV}\right)=45.5$ as, suggests $\beta \simeq 4.48$ which is very close to the analytical result of $9 / 2$ [29].
Comparison between different definitions of phase and time delay is exhibited on an extended energy scale in Fig. 3, where we show the cross section (top), phase shift (middle), and time delay (bottom) in photodetachment of $\mathrm{H}^{-}$( left column) in comparison with analogous calculations for photoionization of $\mathrm{He}$ (right column). We display the phases extracted from the $D$ matrix and the $S$ matrix. In the same panels, we show the HF phase as returned by the frozen core calculation [28].

In the case of $\mathrm{He}$, ground state photoionization to $n_{i}=1$ is indistinguishable from the total experimental cross section [26] indicating that this channel is overwhelmingly dominant. All the three definitions of the phase agree between themselves. This means that the independent electron HF basis represents both the scattering and ionization processes very accurately and correlations are
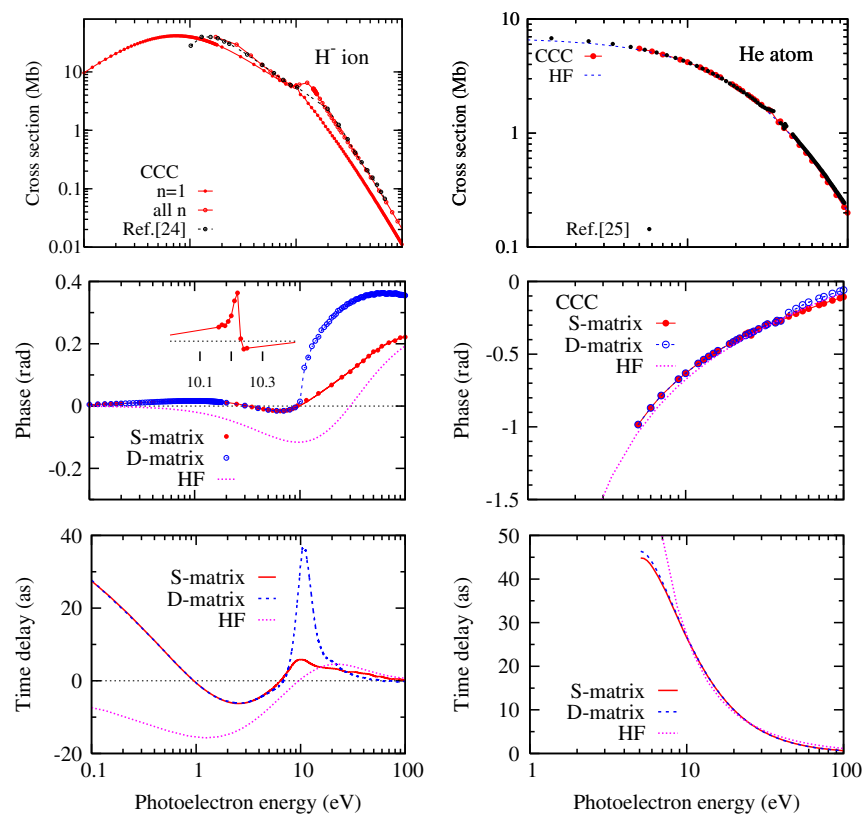

FIG. 3. Left: photodetachment cross sections of $\mathrm{H}^{-}$(top). The partial cross section leaving the $\mathrm{H}$ core in the ground state $n_{i}=1$ and the total single photodetachment cross section summed over $n_{i}<5$ are plotted with filled and open red circles, respectively. The data for the total cross section from Ref. [25] are plotted with open circles. The photoelectron scattering phase (middle) extracted from the $S$ matrix (red filled circles), and the $D$ matrix (open blue circles) are shown in comparison with the frozen-core Hartree-Fock phase (purple dotted line). The red solid and blue dashed lines are cubic spline interpolation of the CCC raw phases. The inset shows the $S$-matrix phase behavior in the vicinity of threshold. Bottom: the Wigner time delay evaluated from the cubic spline interpolation to the $S$-matrix phase (red solid line), the dipole $D$-matrix phase (blue dashed line) and the energy derivative of the HF phase (purple dotted line). Right: Ground state photoionization cross section of $\mathrm{He}$ (top) in comparison with total experimental cross section [26]. The middle and bottom panels are the same as for $\mathrm{H}^{-}$in the left column. 
negligible. Near threshold, the phase exhibits a logarithmic divergence

$$
\sigma_{l}(E) \simeq \eta\left[\ln \sqrt{(l+1)^{2}+\eta^{2}}-1\right] \rightarrow \eta \ln |\eta|,
$$

where $\eta=-1 / \sqrt{2 E}$. The corresponding time delay, shown in the bottom right panel, is always positive and diverges near threshold as $E^{-3 / 2} \ln E$.

The case of $\mathrm{H}^{-}$, visualized in the left column, is manifestly different. The photodetachment to the ground state is still the dominant channel but it only accounts for approximately half of the total cross section above the first excitation threshold. Near the photodetachment threshold, the $D$-matrix phase and $S$-matrix phase are very close but start to deviate visibly above the first excitation threshold. Both phases are very different from the frozen core HF phase indicating a strong core polarization effect. Right on the threshold, the $S$-matrix phase displays a very narrow resonance magnified in the inset of the middle left panel of Fig. 3. This resonance in the dipole singlet channel of the $e-\mathrm{H}$ scattering is related to a quasibound state of the $\mathrm{H}^{-}$ion and is known in the literature (see Sec. 4.3 of [30]). With the exception of this resonance, the phase is continuous across the threshold $\delta_{l}(E)=a_{l}\left(E-E_{t}\right)$ (see Sec. 9.3 of [5]).

The raw CCC phases are cubic spline interpolated and differentiated to obtain the time delay shown in the bottom panel. The HF time delay is obtained by direct numerical differentiation of the HF phase. The CCC time delay below the $n=2$ threshold is very similar in both definitions. Above the threshold, both phases start to grow linearly with the excess energy $E-E_{t}$; however, this growth is manifestly more rapid in the $D$-matrix phase. Accordingly, above the threshold, the photoemission time delay shows a sharp peak reaching the value as large as $\simeq 40$ as, something that can be readily measured. The corresponding peak of the elastic scattering delay is about 10 times smaller. These peak values of the time delay, defined by the coefficients $a_{l}$, can be associated with the switching times of the newly opened photoemission and inelastic scattering channels. Higher above the excitation threshold, the photodetachment delay falls off more rapidly than the elastic scattering delay and becomes slightly negative.

We also applied a more general definition of the time delay in multichannel scattering theory [11], as the diagonal element of the life time matrix

$$
\tau_{1}=\operatorname{Re}\left[i \sum_{n} S_{1 n}\left(d S_{1 n}^{*} / d E\right)\right]=\sum_{n}\left|S_{1 n}\right|^{2} \tau_{1 n},
$$

where $\tau_{1 n}=\operatorname{Im}\left[S_{1 n}^{\prime} / S_{1 n}\right]$. Equation (7) is equivalent to the original definition (1) when $S_{1 n, n \neq 1}=0$. We included three additional inelastic channels $2 s e p, 2 p e s$ and $2 p e d$ to Eq. (7), and the resulting change of the time delay in the elastic $1 s \epsilon p$ channel proved to be insignificant.

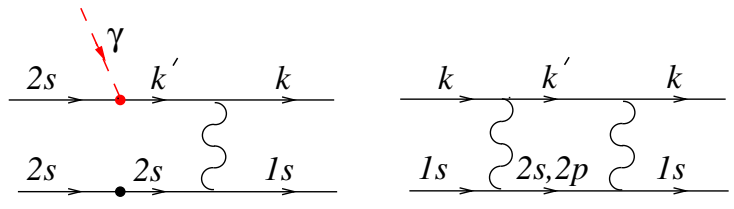

FIG. 4. Graphical representation of the lowest order interelectron interaction in elastic electron scattering (right) and photoemission (left) containing the virtual excitation to the $n=2$ state of the core. The interelectron interaction is shown by a wavy line.

The strong deviation between the time delay in photoemission and electron scattering can be explained by different lowest orders of interelectron interaction mixing the ground state and excitation channels. In elastic electron scattering, the virtual excitation of the hydrogen core to the $n=2$ state requires two successive interactions between the projectile and the target (right diagram of Fig. 4). In photodetachment from $\mathrm{H}^{-}$, it is the interaction in the first order that couples the excitation to the $n=2$ state with the ground state photodetachment (left diagram). This is due to the multiconfiguration expansion of the correlated initial state $\Psi_{0}=\sum_{n l} C_{n l}\left|n l^{2}\right\rangle$, which contains about $20 \%$ of the $2 s^{2}$ configuration. Because of the ground state correlation, the hydrogen core is left with a sufficiently high probability in the $n=2$ excited state. Then, the process requires only one interelectron interaction to relax the core to its ground state. The ground state correlation of this magnitude is absent in $\mathrm{He}$, and hence, there is no threshold enhancement of the time delay in this atom.

In conclusion, we have demonstrated a strong sensitivity of the photoemission time delay to the threshold behavior of the phase which is greatly enhanced in comparison with the photoelectron scattering time delay. As a case study, we considered the two related processes of photodetachment from the $\mathrm{H}^{-}$negative ion and the elastic electron scattering on the hydrogen atom, both leaving this atom in the ground state. We attributed this strong enhancement to the ground state correlation in the initial state of the $\mathrm{H}^{-}$ion. Similar effects will be prominent in photodetachment of the $\mathrm{Li}^{-}$ion near the $2^{2} P$ threshold [31] and photoionization of metastable helium atom near the $n=3$ threshold [18]. In addition, the photoemission time delay will be greatly enhanced near the opening of a deeper photoionization channel. Such a behavior is clearly seen in the photoemission time delay of a valence atomic shell near the threshold of an inner shell in noble gases [32]. In molecules, opening of a new dissociative channel will result in the enhancement of the photoemission time delay in the primary photoionization channel. Such a situation can be observed in many molecules, i.e., $\mathrm{N}_{2}$ (see Figure 3.2.5 of [33]). More generally, phase determination will be particularly useful near special points in the complex energy plane of the system. These are branching points near fragmentation and aggregation thresholds and the conical intersections of two dissociative states of the same symmetry. The latter 
intersections determine the pathways and outcomes of many important photochemical reactions [34,35].

Experimental observation of photoemission time delay near threshold, which reaches values as high as 40 as, can be made by attosecond streaking [6] and interferometric [7] techniques. In negative ions, the measured time delay will not be affected by the laser coupling with the Coulomb field of the remaining ion [36,37]. In neutral species, this coupling will be significantly reduced because the photoelectron energy in the primary channel near a secondary threshold will be large. This permits unhampered measurements of switching times of newly opened photoemission channels and ushers a new field of study of threshold phenomena using attosecond chronoscopy.

Support from the Australian Research Council Grant No. DP120101805 is acknowledged. Resources of the National Computational Infrastructure and the Pawsey Supercomputer Centre were employed.

[1] E.P. Wigner, On the behavior of cross sections near thresholds, Phys. Rev. 73, 1002 (1948).

[2] A. I. Baz', Zh. Eksp. Teor. Fiz. 33, 923 (1958) [The energy dependence of a scattering cross section near the threshold of a reaction, Sov. Phys. JETP 6, 709 (1958)].

[3] H. R. Sadeghpour, J. L. Bohn, M. J. Cavagnero, B. D. Esry, I. I. Fabrikant, J. H. Macek, and A. R. P. Rau, Collisions near threshold in atomic and molecular physics, J. Phys. B 33, R93 (2000).

[4] C. Livermore, C. H. Crouch, R. M. Westervelt, K. L. Campman, and A.C. Gossard, The Coulomb blockade in coupled quantum dots, Science 274, 1332 (1996).

[5] A. Baz', Y. Zel'dovich, and A. Perelomov, Scattering, Reactions and Decay in Nonrelativistic Quantum Mechanics (Israel Program for Scientific Translation, Jerusalem, 1966).

[6] M. Schultze et al., Delay in photoemission, Science 328, 1658 (2010).

[7] K. Klünder et al., Probing Single-Photon Ionization on the Attosecond Time Scale, Phys. Rev. Lett. 106, 143002 (2011).

[8] R. Pazourek, S. Nagele, and J. Burgdörfer, Attosecond chronoscopy of photoemission, Rev. Mod. Phys. 87, 765 (2015).

[9] L. Eisenbud, Ph. D. thesis, Princeton University, 1948.

[10] E. P. Wigner, Lower limit for the energy derivative of the scattering phase shift, Phys. Rev. 98, 145 (1955).

[11] F. T. Smith, Lifetime matrix in collision theory, Phys. Rev. 118, 349 (1960).

[12] A. S. Kheifets and I. A. Ivanov, Delay in Atomic Photoionization, Phys. Rev. Lett. 105, 233002 (2010).

[13] I. Bray and A. T. Stelbovics, Convergent close-coupling calculations of electron-hydrogen scattering, Phys. Rev. A 46, 6995 (1992).

[14] A. S. Kheifets and I. Bray, Photoionization with excitation and double photoionization of helium isoelectronic sequence, Phys. Rev. A 58, 4501 (1998).
[15] I. Bray, D. Fursa, A. Kadyrov, A. Stelbovics, A. Kheifets, and A. Mukhamedzhanov, Electron- and photon-impact atomic ionisation, Phys. Rep. 520, 135 (2012).

[16] A. S. Kheifets and I. Bray, Valence-shell double photoionization of alkaline-earth-metal atoms, Phys. Rev. A 75, 042703 (2007).

[17] A. S. Kheifets, D. V. Fursa, and I. Bray, Two-electron photoionization of ground-state lithium, Phys. Rev. A 80, 063413 (2009).

[18] A. S. Kheifets, A. Ipatov, M. Arifin, and I. Bray, Closecoupling calculations of the two-electron photoionization of the metastable $2^{1,3} S$ helium atom, Phys. Rev. A 62, 052724 (2000).

[19] I. E. McCarthy and A. T. Stelbovics, Momentum-space coupled-channels optical method for electron-atom scattering, Phys. Rev. A 28, 2693 (1983).

[20] M. Venuti and P. Decleva, Convergent multichannel continuum states by a general configuration interaction expansion in a b-spline basis: application to $\mathrm{H}^{-}$photodetachment, J. Phys. B 30, 4839 (1997).

[21] H.-P. Popp and S. Kruse, Negative hydrogen ion detachment cross section from radiation measurements on a PLTE-arc, J. Quant. Spectrosc. Radiat. Transfer 16, 683 (1976).

[22] M. Génévriez and X. Urbain, Animated-beam measurement of the photodetachment cross section of $\mathrm{H}^{-}$, Phys. Rev. A 91, 033403 (2015).

[23] D. Register and R. Poe, Algebraic variational method-a quantitative assessment in $e^{ \pm}-H$ scattering, Phys. Lett. 51A, 431 (1975).

[24] M.-K. Chen, C. D. Lin, and J.Z. Tang, Hyperspherical close-coupling calculation of electron-hydrogen scattering cross sections, Phys. Rev. A 56, 2435 (1997).

[25] J. T. Broad and W. P. Reinhardt, One- and two-electron photoejectrion from $\mathrm{H}^{-}$: A multichannel $j$ matrix calculation, Phys. Rev. A 14, 2159 (1976).

[26] J. A. R. Samson, Z. X. He, L. Yin, and G. N. Haddad, Precision measurements of the absolute photoionization cross sections of He, J. Phys. B 27, 887 (1994).

[27] L. D. Landau and E. M. Lifshitz, in Quantum Mechanics (Non-Relativistic Theory), Course of Theoretical Physics Vol. 3 (Pergamon Press, Oxford, 1985).

[28] L. V. Chernysheva, N. A. Cherepkov, and V. Radojevic, Frozen core Hartree-Fock programm for atomic discrete and continuous states, Comput. Phys. Commun. 18, 87 (1979).

[29] U. Fano and A. R. P. Rau, Atomic Collisions and Spectra (Academic Press, Orlando, 1986).

[30] H. Friedrich, Theoretical Atomic Physics (Springer, Berlin, 2012).

[31] J. Dellwo, Y. Liu, D. J. Pegg, and G. D. Alton, Nearthreshold photodetachment of the $\mathrm{Li}^{-}$ion, Phys. Rev. A 45, 1544 (1992).

[32] A. Ganesan, S. Saha, P. C. Decshmukh, S. T. Manson, and A. S. Kheifets, Electron correlation effects on photoionization time delay in atomic Ar and Xe, in Program of the 2016 Meeting of the Division of Atomic, Molecular, and Optical Physics: Providence, Rhode Island, 2016, http://meetings .aps.org/Meeting/DAMOP16/Session/K1.34.

[33] J. Berkowitz, Photoabsorption, Photoionization, and Photoelectron Spectroscopy (Elsevier Science, New York, 2012). 
[34] M. Kowalewski, K. Bennett, K. E. Dorfman, and S. Mukamel, Catching Conical Intersections in the Act: Monitoring Transient Electronic Coherences by Attosecond Stimulated X-ray Raman Signals, Phys. Rev. Lett. 115, 193003 (2015).

[35] H. S. You, S. Han, J.-H. Yoon, J. S. Lim, and J. Lee, Structure and dynamic role of conical intersections in the $\pi \sigma^{*}$-mediated photodissociation reactions, Int. Rev. Phys. Chem. 34, 429 (2015).
[36] J. M. Dahlström, D. Guénot, K. Klünder, M. Gisselbrecht, J. Mauritsson, A. L'Huillier, A. Maquet, and R. Taïeb, Theory of attosecond delays in laser-assisted photoionization, Chem. Phys. 414, 53 (2013).

[37] R. Pazourek, S. Nagele, and J. Burgdorfer, Time-resolved photoemission on the attosecond scale: opportunities and challenges, Faraday Discuss. 163, 353 (2013). 